\title{
SMF-POLOPT: An Adaptive Multi-temporal Pol(DIn)SAR Filtering and Phase Optimization Algorithm for PSI Applications
}

\author{
Feng Zhao, Student Member, IEEE, and Jordi J. Mallorqui, Senior Member, IEEE
}

\begin{abstract}
Speckle noise and decorrelation can hamper the application and interpretation of PolSAR images. In this paper, a new adaptive multi-temporal Pol(DIn)SAR filtering and phase optimization algorithm is proposed to address these limitations. This algorithm first categorizes and adaptively filters permanent scatterer (PS) and distributed scatterer (DS) pixels according to their polarimetric scattering mechanisms (i.e. the ScatteringMechanism based Filtering (SMF)). Then two different POLDInSAR phase OPTimization methods are applied separately on the filtered PS and DS pixels (i.e. POLOPT). Finally, an inclusive pixel selection approach is used to identify high quality pixels for ground deformation estimation. 31 full-polarization Radarsat-2 SAR images over Barcelona (Spain) and 31 dual-polarization TerraSAR-X images over Murcia (Spain) have been used to evaluate the performance of the proposed algorithm. The PolSAR filtering results show that the speckle of PolSAR images has been well reduced with the preservation of details by the proposed SMF. The obtained ground deformation monitoring results have shown significant improvements, about $\times \mathbf{7 . 2}$ (the fullpolarization case) and $\times 3.8$ (the dual-polarization case) w.r.t. the classical full-resolution single-pol amplitude dispersion method, on the valid pixels' densities. The excellent PolSAR filtering and ground deformation monitoring results achieved by the adaptive Pol(DIn)SAR filtering and phase optimization algorithm (i.e. the SMF-POLOPT) have validated the effectiveness of this proposed scheme.
\end{abstract}

Index Terms-Persistent scatterer interferometry (PSI), polarimetry, ground deformation, speckle filtering, interferometric phase optimization, pixel density.

\section{INTRODUCTION}

D UE to its high accuracy and efficiency, persistent scatterer interferometry (PSI) with synthetic aperture radar (SAR), also known as Differential SAR Interferometry (DInSAR), have been routinely used in terrain and infrastructures motion detection and management. As PSI techniques can only exploit those pixels presenting good phase quality along time, i.e. barely decorrelated, a pixel selection is compulsory prior to the processing of data. These pixels are known as Persistent Scatterers (PSs). The quality of pixels along the data set has to

Manuscript received $* * * * * *$, ****; revised $* * * * * *, * * * *$. This work was supported by the China Scholarship Council (Grant 201606420041), the Spanish Ministry of Economy, Industry and Competitiveness (MINECO), the State Research Agency (AEI) and the European Funds for Regional Development (EFRD) under project TEC2017-85244-C2-2-P, by the National Natura Science Foundation of China (Grant 51574221, 41874044). CommSensLab is Unidad de Excelencia Maria de Maeztu MDM-2016-0600 financed by the State Research Agency (AEI), Spain.

Feng Zhao and J. J. Mallorqui are with the CommSensLab, Universitat Politècnica de Catalunya, 08034 Barcelona, Spain (e-mail: feng.zhao@tsc.upc.edu; mallorqui@tsc.upc.edu). be estimated using a particular metric or measurement from the data to be processed themselves. Basically, according to the scattering mechanisms characterizing their behaviors, pixels (associated with targets present in the scene) can be classified into two general categories: deterministic or permanent scatterers and distributed scatterers. So, the selection criterion has to be adapted to the kind of scatterer to be detected.

The first category of the selection criteria considers deterministic scatterers, also known as Permanent Scatterers (PSs), which are time-invariant and spatially concentrated [1]. These scatterers are slightly affected by decorrelation phenomena and despite they can be generated by natural structures they are more common in urbanized areas. PSI techniques taking advantage of PSs usually work at pixel level and thus the resolution of the single look complex (SLC) images is preserved [1]-[4]. For instance, the classical method of Dispersion of Amplitude $\left(D_{A}\right)$ estimates the phase quality of pixels from their amplitude stability along the SLCs' data set. They conduct well in urban or rocky areas where PSs are more likely to be found. On the contrary, in areas where distributed scatterers (DSs) were the majority its performance would be affected.

Compared with the first category, the selection criteria adapted to DSs usually work over multilooked interferograms and, consequently, at lower resolutions [5]-[7]. Multilooking is applied to reduce the speckle noise and estimate the phase quality of the reduced resolution pixels trough their coherence stability along the intereferograms' stack. On the one side, multilooking improves the phase quality by reducing the phase noise and thus allowing to obtain more reliable results. On the other side, it reduces the resolution and key details can be lost.

More advanced PSI techniques, such as SqueeSAR and its variants, have been proposed to jointly process PS and DS pixels [8], [9]. These techniques usually utilize similarity tests [10], e.g. Kolmogorov-Smirnov (KS) or Anderson-Darling (AD), to search for Spatial Homogeneous Pixels (SHPs) and perform an adaptive filtering based on the SHPs found. Another advanced algorithm is CAESAR [11], which tries to separate different scattering mechanisms within one pixel by decomposing the pixel's covariance matrix using a principal component analysis. Thus, it is able to mitigate the effects of layover in urban areas for PS pixels and reduce decorrelation of DS pixels [11].

PSI techniques have been traditionally applied to singlepolarization data, mainly due to the shortage of longterm polarimetric SAR (PolSAR) datasets. As more satellite 
sensors with polarimetric capabilities were launched, such as RADARSAT-2, TerraSAR-X, Advanced Land Observing Satellite (ALOS-2) or Sentinel-1, the possibility of extending PSI to the polarimetric case became a reality. Therefore, the polarimetric DInSAR (PolDInSAR), or polarimetric PSI (PolPSI), was introduced [12] and developed to improve the detection and characterization of deformation phenomena by increasing the density and quality of valid pixels in comparison with the single polarization case. Starting from the so-called Best method [12], which selects the polarimetric channel with the highest quality estimator among all available channels, PolPSI techniques have been evolved to more sophisticated methods that search the optimal polarimetric channel in a more extended space [13]-[23].

Conventional PolPSI techniques improve interferograms through either the optimization of an amplitude-based criterion (e.g. $D_{A}$, suitable for PSs) [13]-[15], [23], [24] or the maximization of the coherence stability ( $\gamma$, suitable for DSs) [13][15], [20]-[22], [25]-[28]. Another phase quality metric, the temporal sublook coherence (TSC) [29], [30], has also been proposed to optimize interferograms [30], and it is effective mainly for point-like scatterers (i.e. PSs). More recently, by using the phase-based criterion temporal coherence [3], Sadeghi et al. [31] proposed a new PolPSI algorithm. This algorithm is able to retain the full spatial resolution of SLCs, and it is more applicable than the amplitude-based PolPSI approaches in vegetated areas. Nevertheless, it works mainly for PSs and the computational cost of optimizing the temporal coherence is much higher [31].

As PSs and DSs are present in real scenarios more adaptive PolDInSAR optimization algorithms have been proposed to deal simultaneously with both. Inspired by SqueeSAR [8], Navarro-Sanchez proposed an adaptive PolDInSAR optimization method, which is based on a spatial adaptive speckle filtering approach that can jointly process DS and PS pixels [32]. As expected, this new method can achieve higher pixel densities than the conventional ones. However, as it has to carry out a similarity test to identify polarimetric homogeneous pixels (PHPs) [32] for each pixel, its computation burden is high.

In this paper, we propose an alternative adaptive Pol(DIn)SAR optimization algorithm that avoids the timeconsuming similarity test for PSI applications. This algorithm is mainly based on the extension of Lee's PolSAR filter [33] and polarimetric optimization techniques [15], [32]. It first utilizes PolSAR classification results and pixels' scattering mechanisms to identify for each pixel its polarimetric homogeneous pixels (PHPs) [32] and classify it as DS or PS. Then, DS pixels are filtered with the Minimum Mean Square Error (MMSE) method [34] based on their associated PHPs, while PS pixels are preserved with no further modifications. After that, DS and PS pixels are optimized based on their coherence, $\gamma$, and dispersion of amplitude, $D_{A}$, respectively. Finally, the optimized DS and PS pixels are jointly processed to estimate the deformation.

To validate the feasibility and evaluate the performance of the proposed algorithm, it has been tested with two different data sets. One with quad-pol Radarsat-2 data acquired over Barcelona airport (Spain) and the other with dual-pol TerraSAR-X data acquired over Murcia (Spain). Both scenarios are affected by subsidence phenomena. The benefits of the proposed approach in terms of phase quality improvement and higher pixel densities of the final deformation maps have been assessed and discussed.

The paper is organized as follows. Section II describes in detail the proposed adaptive Pol(DIn)SAR filtering and optimization algorithm. In Section III, the data sets of the two sites are briefly described. Then, the deformation monitoring results obtained by the proposed and other classical methods are presented and compared in Section IV. Finally, in Sections $\mathrm{V}$ and VI results are discussed and conclusions are made.

\section{Methods}

\section{A. Scattering-Mechanism based Filtering (SMF)}

The filtering strategy is inspired by the filter proposed by Lee in [33], which was designed for single PolSAR images. In this paper, the filter has been extended to the multi-temporal case and improved to better utilize the rich information within the stack of PolSAR images. As the principle of the proposed filtering is based on pixels' scattering mechanism, it is thus named as the Scattering-Mechanism based Filtering (SMF). It is worth to be mentioned that the selected filter [33] is not the only option to implement the SMF, for instance [35] or any other advanced PolSAR filter could be used instead. In any case, the selected filter should provide a good balance between filter performance and computational efficiency as the PSI approach requires processing large number of images. Fig. 1 shows the scheme of SMF.

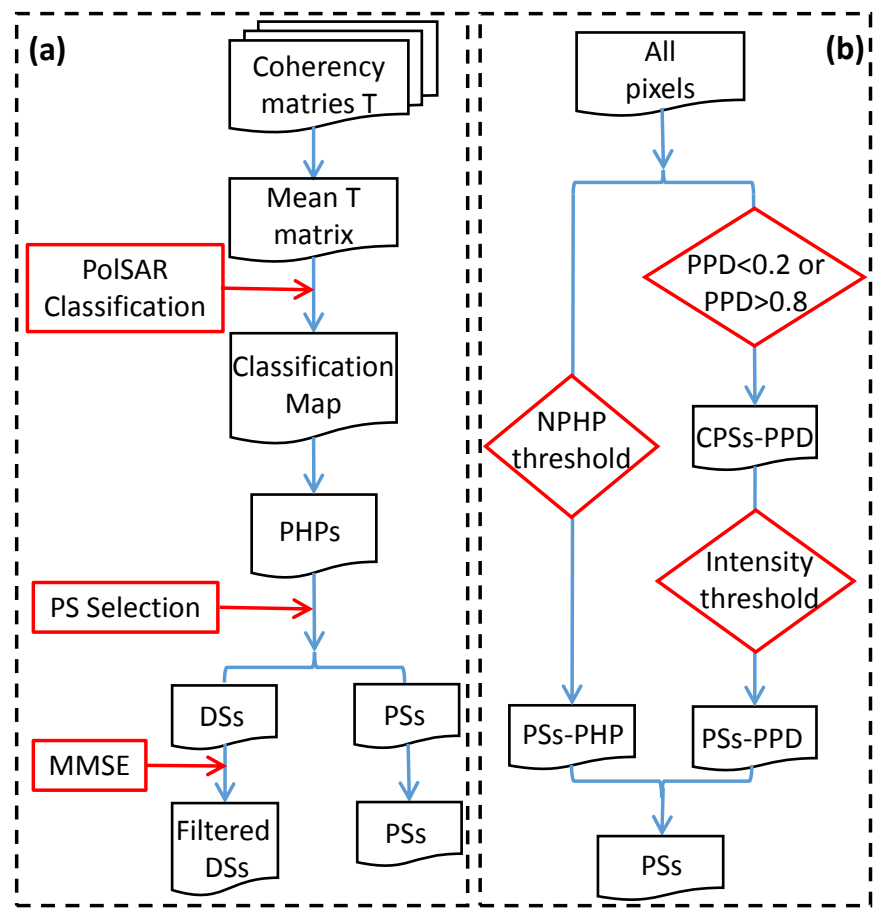

Fig. 1. The scheme of the proposed adaptive multi-temporal Pol(DIn)SAR filtering, i.e. SMF. (a) the overall scheme of the adaptive filter; (b) the subscheme of PS pixel selection. 
1) Identification of Polarimetric Homogeneous Pixels (PHPS): As the proposed filtering scheme is based on PHPs of each pixel, the identification of PHPs is the first step. The PHPs' identification is based on the results obtained from the H/A/Alpha-Wishart PolSAR classifier [36]-[39]. This classifier requires the calculation of the time-series mean coherency matrix $\bar{T}(1)$,

$$
\overline{\boldsymbol{T}}=\frac{1}{N} \sum_{i=1}^{N} \boldsymbol{k}_{i} \cdot \boldsymbol{k}_{i}^{\dagger}
$$

where $N$ is the number of acquisitions, $\boldsymbol{k}_{i}$ is the Pauli vector of the $i$ th acquisition and $\dagger$ represents the Hermitian or conjugate transpose. For quad-pol SAR data, $\boldsymbol{k}_{i}$ can be obtained with (2)

$$
\boldsymbol{k}_{i}=\frac{1}{\sqrt{2}}\left[S_{h h, i}+S_{v v, i}, S_{h h, i}-S_{v v, i}, 2 S_{h v, i}\right]^{T}
$$

where $T$ means the transpose. $S_{h h, i}$ and $S_{v v, i}$ stand for the horizontal and vertical co-polar channels, respectively, and $S_{h v, i}$ is the cross-polar channel of the scattering matrix. If the data is dual-pol, (2) has to be replaced by (3) if only the co-polar channels are available,

$$
\boldsymbol{k}_{i}=\frac{1}{\sqrt{2}}\left[S_{h h, i}+S_{v v, i}, S_{h h, i}-S_{v v, i}\right]^{T}
$$

or by (4) if a co-polar $x x$ and the cross-polar channels are available,

$$
\boldsymbol{k}_{i}=\left[S_{x x, i}, 2 S_{h v, i}\right]^{T} .
$$

Once $\overline{\boldsymbol{T}}$ has been obtained, the H/A/Alpha-Wishart classifier [36]-[39] is used to classify all pixels into 16 categories. The interpretation of the physical characteristics of each category can be found in [37], [39]. After that, a neighboring window $(15 \times 15$ in this paper) centred at each pixel (central pixel) is set to search for its neighboring PHPs. Those pixels that belong to the same category as the central pixel are recognized as PHPs of it.

As an example, the classification results obtained from 31 full-polarization Radarsat-2 images over Barcelona airport, which will be introduced later in Section III, is shown in Fig. 2(c). Observing Fig. 2(c) and the corresponding optical (Fig. 2(a)) and composite RGB (Fig. 2(b)) images of the area, it can be found that the different classes in the area are well segmented. For instance, the runways, corresponding to the class 06 in Fig. 2(c), are perfectly delimited. The numbers of identified PHPs (NPHPs) for each pixel are depicted in Fig. 2(d). As expected and according to the optical and RGB composite images, large NPHPs are concentrated in homogeneous areas like the sea, some fields, roads and runways; while the small ones are associated with heterogeneous areas like the airport terminal, air traffic control tower and buildings.

2) Classification of DS and PS Pixels: After the identification of PHPs, pixels can be classified into DSs and PSs using two complementary methods, one based on the number of PHPs (NPHPs) associated with each pixel and the other the PS pixel selection method presented in [40].

For the former, a threshold on the NPHPs is set mainly considering SAR images' resolution and characteristics of the study areas. Concretely, this threshold should be high if

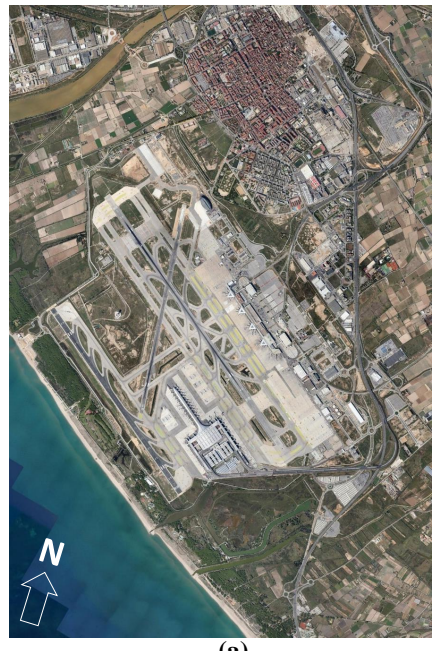

(a)

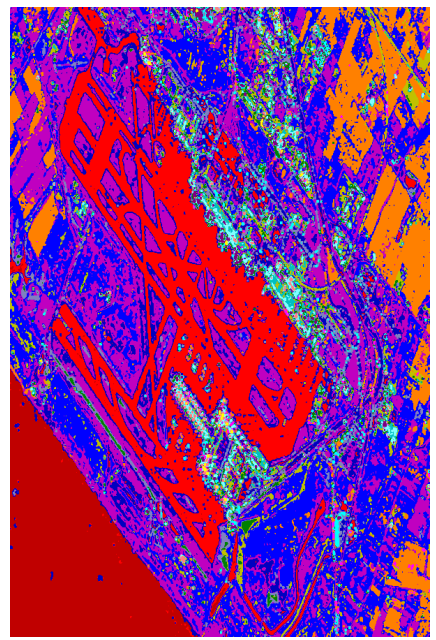

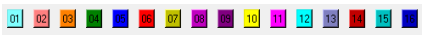

(c)

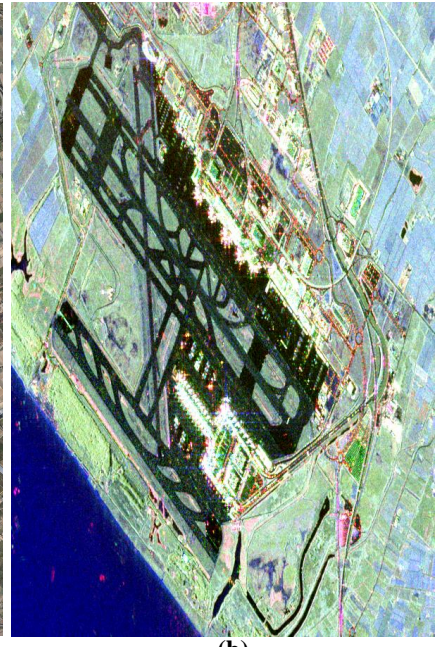

(b)

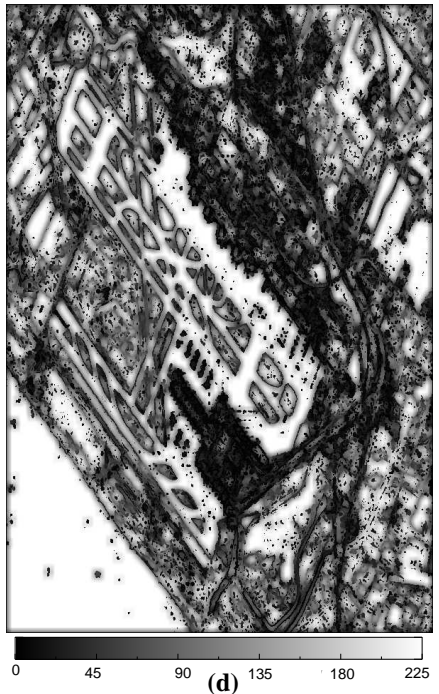

Fig. 2. (a) The GoogleEarth image over the Barcelona airport, Spain, which has been rotated and flipped to better match SLC orientation; (b) the RGB composite image of the area, formed by SAR images' temporal average intensities. The color coding is $\mathrm{R}=|H H-V V|, \mathrm{G}=2|H V|$, and $\mathrm{B}=$ $|H H+V V|$; (c) the multi-temporal PolSAR data classification result over the area; (d) the number of identified PHPs for each pixel based on (c) with a $15 \times 15$ neighboring window.

working with high resolution images or homogeneous study scenes; while it should be low for the opposite cases (low resolution images or heterogeneous scenes). It is worth to be mentioned that other methods like the one used in SqueeSAR [8] could be adopted to determine the proper threshold. In this paper, after considering the two above-mentioned factors and testing a few threshold candidates over the two test sites, the threshold has been set to 9. Pixels with NPHPs lower than the threshold are considered as PSs (PSs-PHP in Fig. 1(b)).

For the latter, PSs can be selected based on the normalized polarization phase difference (PPD) and the pixel mean intensity. The PPD can be calculated for each pixel of the scene considering the whole dataset with (5),

$$
\chi=\frac{\sum_{i=1}^{N}\left|\phi_{H H}^{i}-\phi_{V V}^{i}\right|}{N \pi}
$$

where $N$ is the number of acquisitions, $\phi_{H H}^{i}$ and $\phi_{V V}^{i}$ are 
for the $i$ th acquisition the phases of the co-polar channels [40]. Then those pixels with PPDs below 0.2 or above 0.8 are identified as the candidates of PSs-PPD (CPSs-PPD in Fig. 1(b)) [40]. In the next step, a threshold on pixels' temporal averaged intensities is set to recognize the PSs-PPD from the CPSs-PPD, as Fig. 1(b) shows. By following [40], two times of the mean value of all pixels' temporal averaged intensities is set as the threshold in this paper. And then those CPSs-PPD with temporal averaged intensities greater than this threshold are identified as PSs-PPD.

Finally, the pixels selected by both methods, PSs-PHP and PSs-PPD, are combined to obtain the final selection of PSs, and the rest of pixels are considered as DSs. In other words, a pixel is recognized as PS if it is selected by any of the two PS pixel selection methods. The block diagram of PS and DS pixels' classification (or PS pixel selection) are illustrated in Fig. 1(b). It has to be noted that the complementary strategy using PPD can only be applied if both co-polar polarizations are available. So, for the dual-pol case where only one copolar channel is available only PSs-PHP can be obtained and treated as the final selection of PSs.

3) Pol(In)SAR Filtering: The filtering strategies are adapted to the two types of scatterers in which the pixels of the images have been classified, as Fig. 1(a) shows. As the signal to noise ratio (SNR) of PSs is supposed to be high and the resolution has to be preserved, no filtering is applied on them. For DSs, the Minimum Mean Square Error (MMSE) filter [33], [34] has been applied to better preserve features and edge sharpness. Equation (6) shows the filtering strategy,

$$
\boldsymbol{T}_{\text {filt }}=\boldsymbol{T}_{\text {mean }}+b\left(\boldsymbol{T}_{\text {ori }}-\boldsymbol{T}_{\text {mean }}\right)
$$

where $\boldsymbol{T}_{\text {filt }}$ and $\boldsymbol{T}_{\text {ori }}$ are the filtered and original $\boldsymbol{T}$ matrices of the DS central pixel, respectively. $\boldsymbol{T}_{\text {mean }}$ is the averaged $\boldsymbol{T}$ matrices of the central pixel and all its associated PHPs. $b$ is a weighting factor ranging from 0 to 1 , which is estimated by minimizing the mean square error of the $\boldsymbol{T}$ matrices' spans of the central pixel and its PHPs. A detailed description on how to compute the weighting factor $b$ can be found in [34], [39]. It has to be noted that, for PolSAR filtering, the spans of the PolSAR coherency matrices are used for the estimation of $b$; while for PolInSAR filtering, the spans of the PolInSAR coherency matrices should be used instead.

One PolSAR image over Barcelona airport has been used to illustrate the effectiveness of the SMF adaptive filter. Besides SMF, the PolSAR image has been also filtered with another two well established filters, i.e the Lee's scattering-modelbased speckle filtering (Lee SM) [33] and the intensitydriven adaptive-neighborhood filtering (IDAN) [41], in order to compare their performances. The results are presented in Fig. 3, where the original PolSAR image is also shown to visualize the noise reduction each filter is able to achieve. All the three filters have reduced the PolSAR image's speckle noise, however the proposed SMF strategy can better preserve the details of structures while the other two tend to blur their edges.

\section{B. Adaptive PolDInSAR Phase Optimization and Pixel Selec-} tion

1) Adaptive PolDInSAR Phase Optimization Algorithm: The PolInSAR vector $\boldsymbol{K}$ between two polarimetric acquisitions can be expressed as (7)

$$
K=\left[k_{1}, k_{2}\right]
$$

where the Pauli vector $\boldsymbol{k}_{i}(i=1,2)$ can be derived from equations (2) and (3) or (4) for the quad- or dual-pol, respectively [42]. Mathematically, $\boldsymbol{k}_{i}$ can be projected onto an unitary vector $\boldsymbol{\omega}_{\boldsymbol{i}}$ to obtain a generic scattering coefficient $\boldsymbol{\mu}_{i}=\boldsymbol{\omega}_{\boldsymbol{i}}^{\dagger} \cdot \boldsymbol{k}_{i}$. The complex $\boldsymbol{\mu}_{i}$ is analogous to a new single-pol SAR image obtained from a pixel level linear combination, determined by the projection vector $\omega_{i}$, of the original scattering matrices. In PolDInSAR applications the same projection vector has to be used for all images. If not, the choice of different projections vectors for each image of the interferograms may lead to undesired changes in the phase centers of the scatterers [15]. So, it has to be ensured for any pixel that $\omega_{1}=\omega_{2}=\omega$ for all images of the data set.

For point-like scatterers, PSs, $\boldsymbol{K}$ is deterministic. The amplitude dispersion index $D_{A}$ [1], one common phase quality estimator for PSs, can be generalized to the PolDInSAR case (8),

$$
D_{A}=\frac{\sigma_{A}}{m_{A}}=\frac{1}{\overline{\left|\boldsymbol{\omega}^{\dagger} \boldsymbol{k}\right|}} \sqrt{\frac{1}{N} \sum_{i=1}^{N}\left(\left|\boldsymbol{\omega}^{\dagger} \boldsymbol{k}_{i}\right|-\overline{\left|\boldsymbol{\omega}^{\dagger} \boldsymbol{k}\right|}\right)^{2}}
$$

with

$$
\overline{\left|\boldsymbol{\omega}^{\dagger} \boldsymbol{k}\right|}=\frac{1}{N} \sum_{i=1}^{N}\left|\boldsymbol{\omega}^{\dagger} \boldsymbol{k}_{i}\right|
$$

where $\sigma_{A}$ and $m_{A}$ are the standard deviation and mean of the generic images' amplitudes, $N$ is the number of images, the over line indicates the empirical mean value [13], [15].

In case of distributed scatterers, due to the complex behaviour of the scattering process within each resolution cell, $\boldsymbol{K}$ behaves as a random vector. Thus, under the the hypotheses of spatial homogeneity and ergodicity, the $6 \times 6$ PolInSAR coherency complex matrix $\boldsymbol{T}_{6}$ is used to characterize the scatterer behavior,

$$
\boldsymbol{T}_{6}=E\left\{\boldsymbol{k} \boldsymbol{k}^{\dagger}\right\}=\left[\begin{array}{ll}
\boldsymbol{T}_{11} & \boldsymbol{\Omega}_{12} \\
\boldsymbol{\Omega}_{12}^{\dagger} & \boldsymbol{T}_{22}
\end{array}\right]
$$

where $E$ is the expectation operator, which is usually implemented with a spatial neighboring average. $\boldsymbol{T}_{11}$ and $\boldsymbol{T}_{22}$ are the individual coherency matrices of each PolSAR data set, $\Omega_{12}$ is the PolInSAR coherency matrix [42]. The coherency matrices can be computed as,

$$
\boldsymbol{T}_{i i}=E\left\{\boldsymbol{k}_{i} \boldsymbol{k}_{i}^{\dagger}\right\} \quad \boldsymbol{T}_{j j}=E\left\{\boldsymbol{k}_{j} \boldsymbol{k}_{j}^{\dagger}\right\} \quad \boldsymbol{\Omega}_{i j}=E\left\{\boldsymbol{k}_{i} \boldsymbol{k}_{j}^{\dagger}\right\} .
$$

The generalized polarimetric mean interferometric coherence $\overline{|\gamma|}$ of the inteferogram data set can be obtained from the ones of each available interferogram, $\gamma_{k}(\omega)$,

$$
\overline{|\gamma|}=\frac{1}{K} \sum_{k=1}^{N_{i n t}}\left|\gamma_{k}\right|, \quad \text { with } \gamma_{k}(\boldsymbol{\omega})=\frac{\boldsymbol{\omega}^{\dagger} \boldsymbol{\Omega}_{i j} \boldsymbol{\omega}}{\sqrt{\boldsymbol{\omega}^{\dagger} \boldsymbol{T}_{i i} \boldsymbol{\omega}} \sqrt{\boldsymbol{\omega}^{\dagger} \boldsymbol{T}_{j j} \boldsymbol{\omega}}}
$$



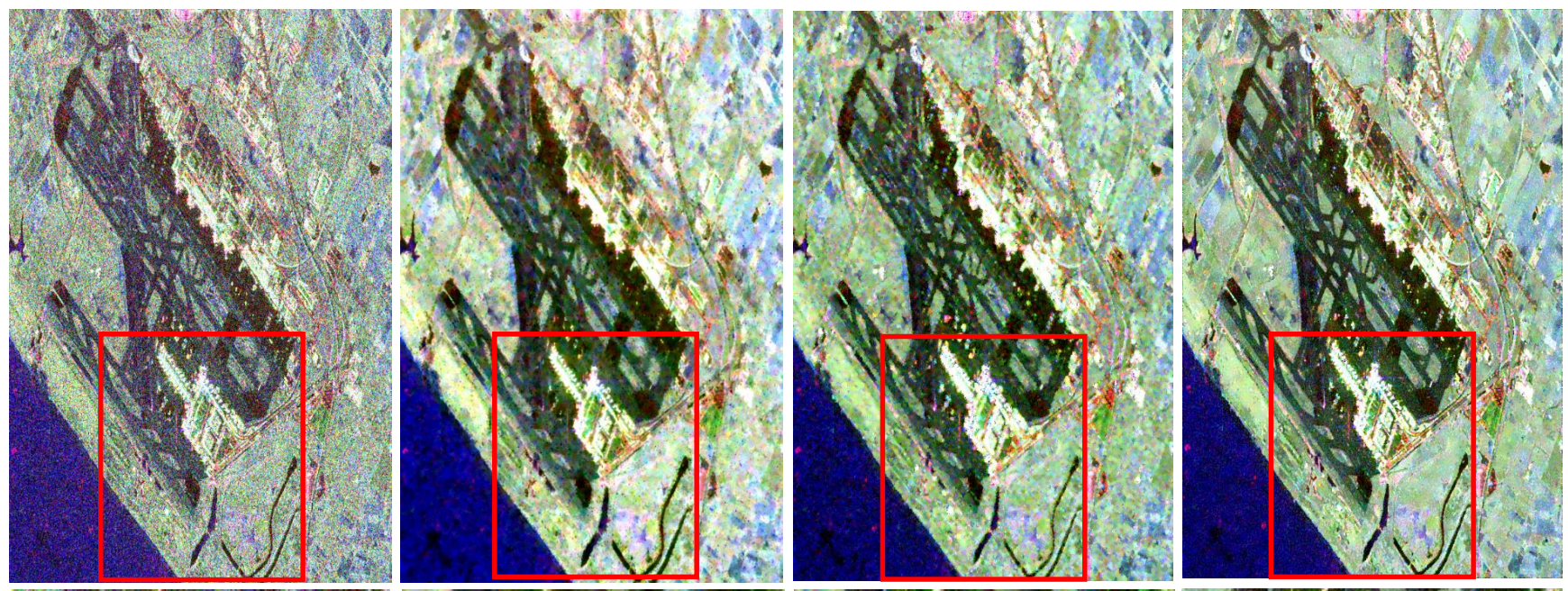

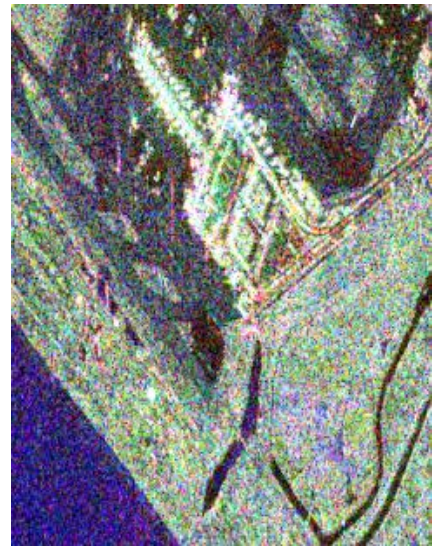

(a) Original

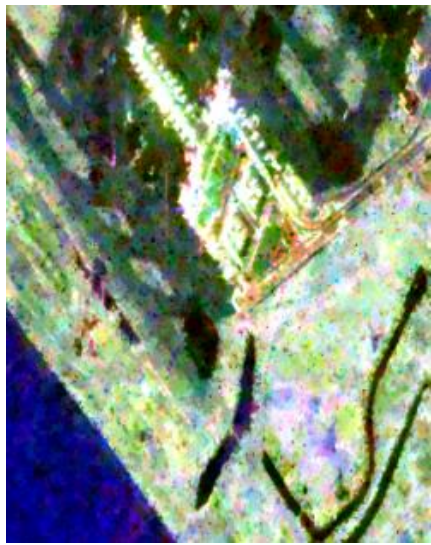

(b) IDAN

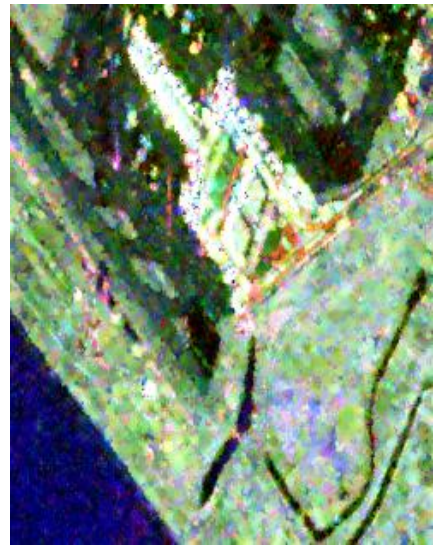

(c) Lee SM

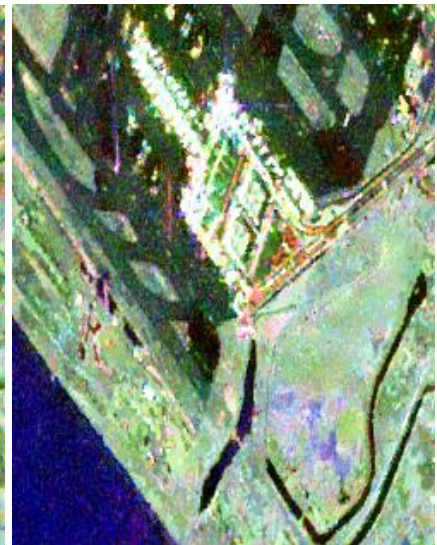

(d) SMF

Fig. 3. Original and filtered Pauli RGB composites over the Barcelona airport. Bottom row shows the close-up of the area limited by the red rectangle in the top row. The color channels are $\mathrm{R}=\mathrm{HH}-\mathrm{VV}, \mathrm{G}=2 \mathrm{HV}$, and $\mathrm{B}=\mathrm{HH}+\mathrm{VV}$.

where $N_{\text {int }}$ is the total number of included interferograms [13], [15].

The objective of the PolDInSAR optimization is to search for the optimal projection vector $\boldsymbol{\omega}$, i.e. the optimal polarimetric channel, that maximizes for each pixel a quality parameter. This is minimizing $D_{A}$ or maximizing $\overline{|\gamma|}$.

The unitary projection vector $\boldsymbol{\omega}$ can be parameterized for quad-pol data as [13], [15],

$$
\boldsymbol{\omega}=\left[\begin{array}{l}
\cos (\alpha) \\
\sin (\alpha) \cos (\beta) e^{j \delta} \\
\sin (\alpha) \sin (\beta) e^{j \psi}
\end{array}\right], \quad\left\{\begin{array}{l}
0 \leq \alpha \leq \pi / 2 \\
0 \leq \beta \leq \pi \\
-\pi \leq \delta<\pi \\
-\pi \leq \psi<\pi
\end{array}\right.
$$

or a simplified version for the dual-pol case,

$$
\boldsymbol{\omega}=\left[\begin{array}{l}
\cos (\alpha) \\
\sin (\alpha) e^{j \psi}
\end{array}\right], \quad\left\{\begin{array}{l}
0 \leq \alpha \leq \pi / 2 \\
-\pi \leq \psi<\pi
\end{array}\right.
$$

The optimization problem is to find the parameters $\alpha, \beta, \delta$ and $\psi$ (or $\alpha$ and $\psi$ ) that optimize the phase quality estimator selected. In this paper, the equal scattering mechanism (ESM) method has been used to perform the optimization [15]. The main advantage of this strategy is that it exploits the whole polarimetric space and thus it is able to find the optimum projection vector $\boldsymbol{\omega}$, but its computational cost can be very high unless optimization strategies are used [15]. Other wellknown strategies like the Best and the Suboptimun Scattering Mechanism (SOM) are detailed in [14], [15].

Traditional PolDInSAR optimization methods optimize all pixels of the whole image either via $D_{A}$, if PSs want to be detected, or $\overline{|\gamma|}$, if the objectives are DSs. The proposed method takes advantage of the classification of the image pixels in PSs and DSs to optimize each one according to its scattering characteristics. As the POLDInSAR OPTimization method is based on the SMF introduced in previous sections, it has been named as SMF-POLOPT. The proposed SMFPOLOPT is more adaptive than the classical algorithms mainly in two aspects. In addition of optimizing each pixel according to its scattering characteristic, the filtering and calculation of the PolInSAR coherency complex matrix $\boldsymbol{T}_{6}$ is optimized for DSs as for each pixel only their similar ones, its PHPs, are used. The latter helps to better filter and reduce the bias of the estimated $\boldsymbol{T}_{6}$ in heterogeneous areas.

2) PolDInSAR Pixel Selection: As two phase quality estimators have been used for the optimization in the SMFPOLOPT scheme, the pixel selection method has to be also based on both $D_{A}$ (for PSs) and $\overline{|\gamma|}$ (for DSs). The method presented in [32] to jointly select high quality pixels from both 
PSs and DSs has been applied. Pixels are selected based on their estimated phase quality, i.e. their phase standard deviation (STD). $D_{A}$ is a good estimator of the pixel's STD for PSs with high signal to noise ratios (SNR) [1]. There is not a mathematical relation between the measured $D_{A}$ and phase STD but it can be easily calculated numerically as a function of the number of images of the data set. Similarly for DSs, the maximum likelihood estimator of the coherence, $\overline{|\gamma|}$, can be mathematically estimated through an spatial averaging of pixels around the pixel of interest. This estimated coherence can be related, depending on the number of looks of the estimation window, with its associated phase STD [43], [44]. Therefore, for both types of pixels the selection threshold is based on the same metric, i.e. the phase STD. All pixels with phase STDs below a given threshold are selected for their subsequent PSI processing.

To calculate the phase STD from $\overline{|\gamma|}$, the equivalent number of looks $E N L$, has to be calculated. $E N L$ is the number of independent samples averaged in the estimation, which can be smaller than the number of averaged pixels when the weighted averaging is applied. Equation (6) can be rewritten as (15),

$$
\begin{array}{r}
\boldsymbol{T}_{6}^{\text {filt }}=\frac{1}{L}\left(\boldsymbol{T}_{6}^{\text {ori }}+\boldsymbol{T}_{6}^{\text {PHPs }}\right) \\
+b\left[\boldsymbol{T}_{6}^{\text {ori }}-\frac{1}{L}\left(\boldsymbol{T}_{6}^{\text {ori }}+\boldsymbol{T}_{6}^{\text {PHPs }}\right)\right] \\
=\frac{(1-b)}{L} \boldsymbol{T}_{6}^{\text {PHPs }}+\frac{1+b(L-1)}{L} \boldsymbol{T}_{6}^{\text {ori }}
\end{array}
$$

with

$$
\boldsymbol{T}_{6}^{P H P s}=\sum_{i=1}^{L-1} \boldsymbol{T}_{6}^{P H P, i}
$$

where $L$ is the number of pixels averaged, i.e. the central pixel and its associated PHPs, $\boldsymbol{T}_{6}^{\text {ori }}$ and $\boldsymbol{T}_{6}^{P H P s}$ represent the central pixel's PolInSAR coherency matrix (unfiltered) and the summation of all its PHPs' PolInSAR coherency matrices, as (16) shows, where $\boldsymbol{T}_{6}^{P H P, i}$ is the $i$ th PHP's PolInSAR coherency matrix.

The equivalent number of looks $E N L$ of the filtered matrix $\boldsymbol{T}_{6}^{\text {filt }}$, can be estimated with (17) [39], [45],

$$
E N L=\operatorname{Var}\left(I_{\text {ori }}\right) / \operatorname{Var}\left(I_{\text {filt }}\right)
$$

where $\operatorname{Var}\left(I_{\text {ori }}\right)$ and $\operatorname{Var}\left(I_{\text {filt }}\right)$ are the variances of the original and filtered intensities, i.e. the variances of any two corresponding elements taken from the diagonals of the $\boldsymbol{T}_{6}^{\text {ori }}$ and $\boldsymbol{T}_{6}^{\text {filt }}$, respectively. The central pixel and its PHPs are assumed independent to each other and with the same intensity variance, i.e. $\operatorname{Var}\left(I_{\text {ori }}\right)=\operatorname{Var}\left(I_{P H P, i}\right)$. Under this assumption, the relationship between $\operatorname{Var}\left(I_{P H P s}\right)$, which is the variance of intensity from $\boldsymbol{T}_{6}^{P H P s}$, and $\operatorname{Var}\left(I_{\text {ori }}\right)$ can be expressed as (18) by referring to (16).

$$
\operatorname{Var}\left(I_{P H P s}\right)=(L-1) \operatorname{Var}\left(I_{P H P, i}\right)=(L-1) \operatorname{Var}\left(I_{\text {ori }}\right)
$$

The filtered intensity variance $\operatorname{Var}\left(I_{\text {filt }}\right)$ can be derived according to the law of variance propagation based on (15) and (18),

$$
\begin{array}{r}
\operatorname{Var}\left(I_{\text {filt }}\right)=\left[\frac{(1-b)}{L}\right]^{2} \operatorname{Var}\left(I_{P H P s}\right)+ \\
{\left[\frac{1+b(L-1)}{L}\right]^{2} \operatorname{Var}\left(I_{\text {ori }}\right)} \\
=\left\{\left[\frac{(1-b)}{L}\right]^{2}(L-1)+\left[\frac{1+b(L-1)}{L}\right]^{2}\right\} \operatorname{Var}\left(I_{\text {ori }}\right)
\end{array}
$$

then substituting (19) into (17), ENL can be calculated as

$$
\begin{aligned}
E N L=1 /\left\{\left[\frac{(1-b)}{L}\right]^{2}(L-1)\right. & \left.+\left[\frac{1+b(L-1)}{L}\right]^{2}\right\} \\
& =L /\left(1+b^{2} L-b^{2}\right)
\end{aligned}
$$

It can be found from (20) that $E N L$ is determined by $L$, the number of pixels participating in the filtering, and the weighting factor $b$.

When $b$ equals to 1 , which means $\boldsymbol{T}_{6}^{\text {filt }}=\boldsymbol{T}_{6}^{\text {Ori }}$ (no filtering is applied) as (15) shows, $E N L$ calculated by (20) is 1 . When $b$ equals to $0, \boldsymbol{T}_{6}^{f i l t}$ is estimated to be $\left(\boldsymbol{T}_{6}^{O r i}+\boldsymbol{T}_{6}^{P H P s}\right) / L$ as (15) shows. In this case, the filtered coherency matrix $\boldsymbol{T}_{6}^{\text {filt }}$ is actually the averaging of all the $L$ independent samples, and $E N L$ calculated by (20) is $L$. The valid values of the estimated $E N L$ in these two special cases (no filtering and averaging cases) indicate the correctness of (20).

\section{DATA SETS}

Two different orbital SAR data sets are employed to evaluate the performance of the proposed algorithm. The RGB Pauli vector composite of both, which are derived by averaging the time-series intensities, are shown by Fig. 2(b) and Fig. 4 , respectively.

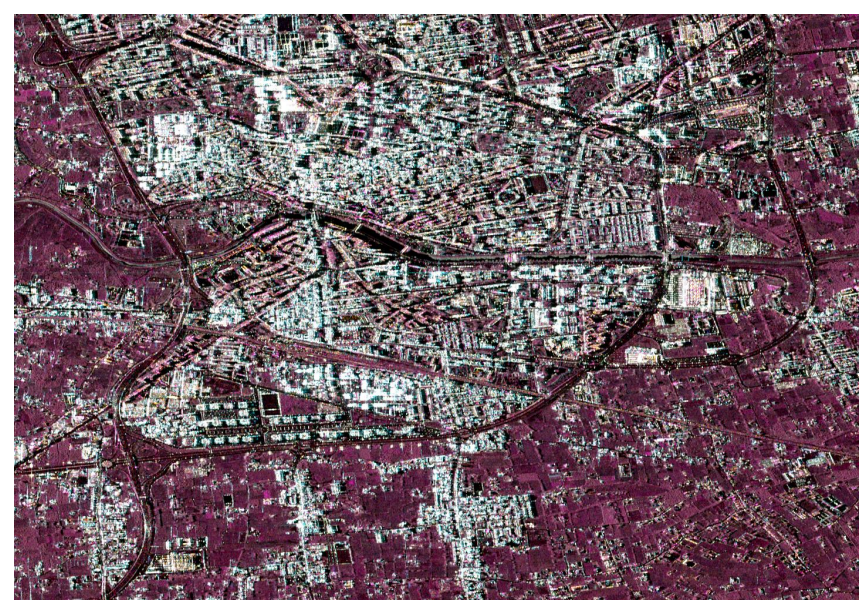

Fig. 4. RGB color composite from Pauli vectors of the Murcia test area. The color coding is $\mathrm{R}=|H H-V V|, \mathrm{G}=2|H H|$, and $\mathrm{B}=|H H+V V|$.

The first data set consists on 31 stripmap full-pol Radarsat2 images acquired from May 2010 to July 2012 over the Barcelona airport, as shown in Fig. 2(b). Radarsat-2 works at $\mathrm{C}$-band and has a revisit period of 24 days. The resolutions of the images are $5.1 \mathrm{~m}$ in azimuth and $4.7 \mathrm{~m}$ in slant-range. The processing has been applied over an area of $902 \times 602$ 
pixels. From Fig. 2(b) we can find that the test site is partially vegetated, which makes it perfect for the assessment of the proposed algorithm in suburban areas.

The other data set consists on 31 dual-pol (HH and VV polarizations) TerraSAR-X images, from February 2009 to February 2010, of Murcia city (located in the south-east of Spain, Fig. 4). This X-band data has a shorter revisit time of only 11 days. The images' resolutions in azimuth and slant-range directions are 2.44 and $0.91 \mathrm{~m}$, respectively. The processed area is $1644 \times 2402$ pixels covering the central and southern parts of the city.

Besides the PolSAR images, two external DEMs from the Spanish Instituto Geografico Nacional (IGN) with $5 \mathrm{~m}$ resolution have also been used to generate the differential interferograms.

\section{RESUlts}

All processing strategies have been integrated into SUBSIDENCE-GUI, UPC's DInSAR processing chain that is the software implementation of the Coherent Pixel Technique (CPT) [6], [7]. In this section, the performance of the proposed algorithms is evaluated in terms of phase optimization and pixels' densities of the derived deformation maps. In order to better illustrate the advantages of the proposed approaches, i.e. the SMF and SMF-POLOPT, they have been compared with other two conventional full resolution methods, i.e. $D_{A}$ (only the HH channel) and ESM- $D_{A}$ (referred hereafter as ESM for the sake of simplicity ). SMF results correspond also to the HH channel.

The complete PSI processing consists mainly in three steps: differential interferograms generation, pixel selection and deformation estimation. During the generation of the interferograms, except for the $D_{A}$ approach, the other three methods filter or optimize DInSAR phases taking advantage of the polarimetric data. Fig. 5 shows the optimized phases with the different methods of a Radarsat- 2 interferogram over Barcelona airport. The unfiltered HH channel's phase is also included for comparison purposes. The ESM method is able to maximize the phase quality mainly on PSs, like in the airport terminal and building areas as seen in Fig. 5(c). The SMFPOLOPT and SMF approaches significantly reduce the phase noise level on DSs, e.g. the runway areas as seen in Fig. 5(a) and Fig. 5(b). As expected, the SMF-POLOPT method shows a better performance than SMF thanks to the further polarimetric optimization of the adaptively filtered interferograms. Good examples of this are the roads highlighted by the black dashed lines in Fig. 5(a). Fig. 6 shows the close-up of the airport terminal section of Fig. 5. As the SMF-POLOPT can adaptively optimize both PSs and DSs, it presents the best performance among all methods.

After generating the differential interferograms, those pixels with phase qualities over a given threshold are selected. For $D_{A}$ and ESM method, pixels with $D_{A}$ values below 0.25 are selected. The pixel selection strategies for the SMF-POLOPT and SMF approaches are identical and they follow the method introduced in the previous section. To make a fair comparison, the same phase standard deviation threshold (around $15^{\circ}$ ) is used for all cases during pixel selection. Finally, based on the selected pixels and generated interferograms, the CPT algorithm is used to estimate the deformation.

For Barcelona Airport full-pol Radarsat-2 data, the retrieved deformation velocity maps are shown in Fig. 7. Very similar subsidence trends are detected by all four approaches, which illustrates the goodness of the proposed algorithms. The subsidence areas are mainly located at the airport access road and terminal $\mathrm{T} 1$ areas, and the maximum subsidence velocity reaches up to $2.5 \mathrm{~cm} /$ year. The deformation amount and patterns are in good accordance with previous studies [14], [15], [32], which further validates the reliability of the results.

As Fig. 7 shows, all the three advanced algorithms perform better, in terms of pixels' densities, than the classical single-pol $D_{A}$ method. The density improvement of ESM with respect to single-pol $D_{A}$ is around $\times 2.7(11,248$ compared to 4,149$)$. The improvement is mostly due to the polarimetric optimization provided by ESM as the three polarimetric channels are combined into a single one. The SMF method applied to $\mathrm{HH}$ channel achieves an increase of pixels of around $\times 1.8$ w.r.t. single-pol $D_{A}$ (7,502 versus 4,149$)$. This improvement is due to the inclusive pixel filtering and selection strategy in SMF, which is able to reduce DS pixels' noise and identify high quality pixels from both PS, over buildings, and DS, over runaways. As expected, the highest pixel densities are obtained by SMF-POLOPT, as it combines the benefits of the adaptive filtering with the polarimetric optimization of data. The increase of SMF-POLOPT w.r.t. single-pol $D_{A}$ is around $\times 7.2(29,963$ compared to 4,149$)$. The highest pixel density allows SMF-POLOPT to better detect and characterize the extend and details of ground motion than the other methods.

The estimated ground deformation results of Murcia with dual-pol TerraSAR-X data are shown in Fig. 8. Significant subsidence phenomena are detected at the south-east part of the city by all methods with a maximum subsidence velocity up to $2.5 \mathrm{~cm} / \mathrm{year}$.

Similarly with the full-pol data, all the advanced methods have achieved improvements on the numbers of selected pixels compared with the single-pol $D_{A}$ method. Particularly, the number of pixels with single-pol $D_{A}$ is 162,513 , and the counterparts of ESM, SMF and SMF-POLOPT are 385,407 $(\times 2.4), 278,522(\times 1.7)$ and $613,995(\times 3.8)$, respectively. The SMF-POLOPT presents again the best performance and SMF outperforms the single-pol $D_{A}$ method in terms of pixel density, proving the effectiveness of the proposed algorithms also on dual-pol SAR data.

Detailed views of two subsidence areas of Murcia are shown in Fig. 9 and Fig. 10 to compare all four algorithms' capability of monitoring small-scale ground motion. The location of Fig. 9 is highlighted by the white rectangle in Fig. 8(a), which corresponds to a highway crossing north to south. In this small area, deformations are detected on the access ramps of a bridge, but the bridge itself is stable (the red rectangle in Fig. 9(a)). The access ramps do not have such deep and strong foundations as the bridge ones, so they are subsiding while the deck not. It can be seen that this deformation phenomenon is better revealed by the SMF and SMF-POLOPT results than that of single-pol $D_{A}$ or ESM approaches, which is owed 


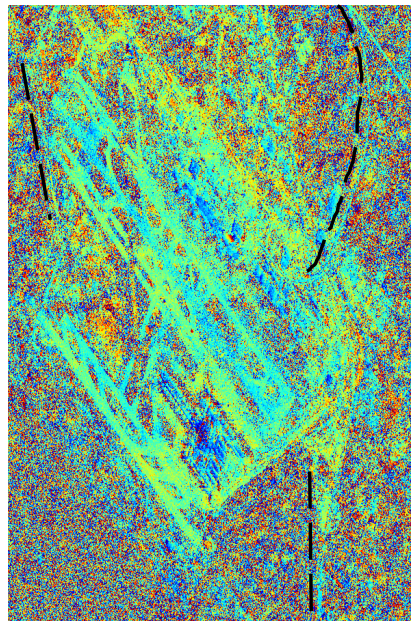

(a) SMF-POLOPT

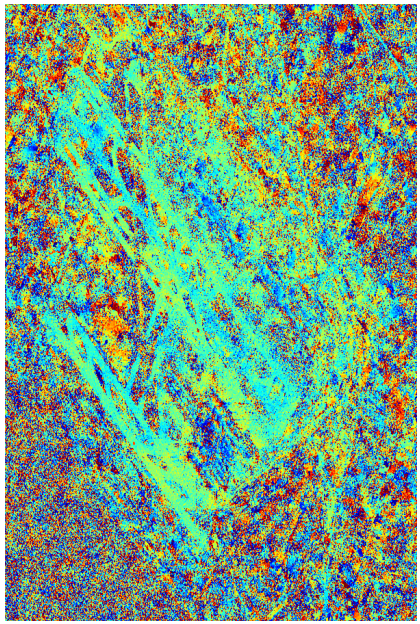

(b) SMF

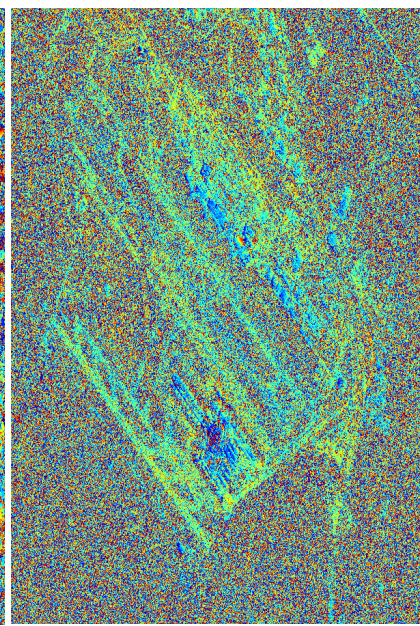

(c) ESM

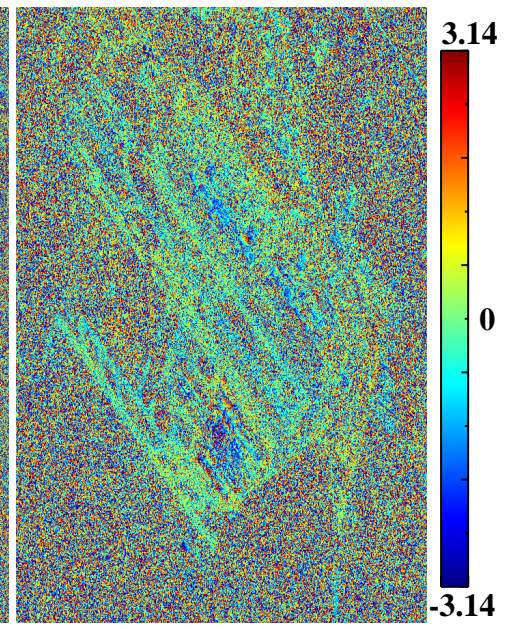

(d) $\mathbf{H H}$

Fig. 5. An interferogram phase optimization results of Barcelona airport by different algorithms. The black dashed lines in (a) indicate the locations of roads where the SMF-POLOPT approach presents the best optimization effect.

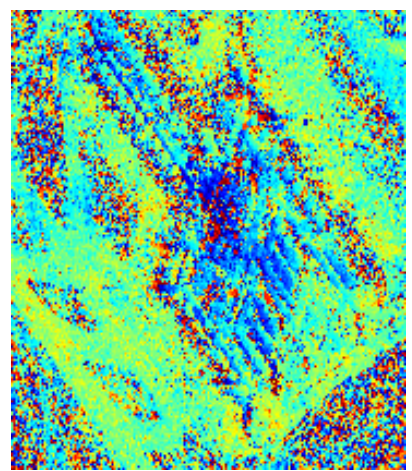

(a) SMF-POLOPT

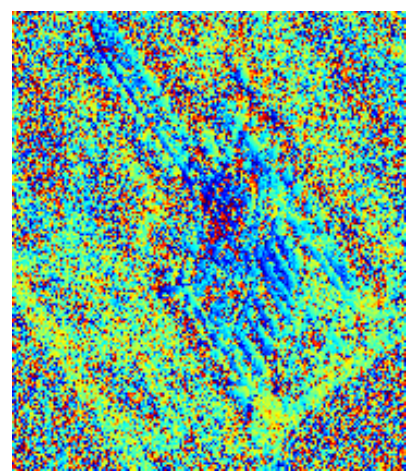

(c) ESM

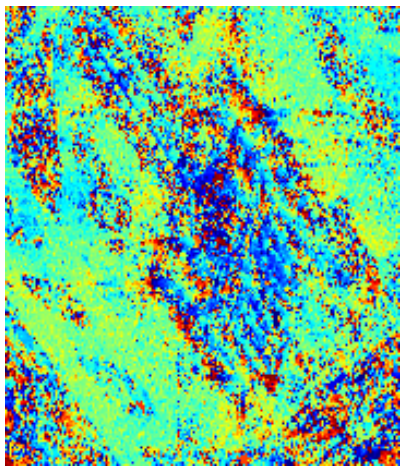

(b) SMF

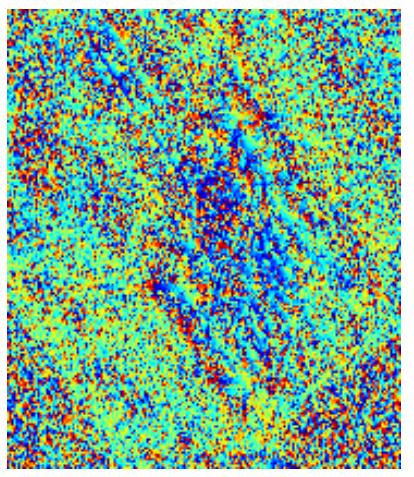

(d) $\mathbf{H H}$
Fig. 6. Close-up of the phase optimization results of Fig 5, which corresponds to the area of the airport terminal.

to the higher pixels' densities provided by their capability to select DS pixels over the roads.

The other detailed view is located around the southern highway bridge area, which has been highlighted by the red rectangle in Fig. 8(a). PS pixels (e.g. the buildings) and DS pixels (e.g. the highway) coexist simultaneously within this area, and we can find from Fig. 10(b) and (c) that the ESM and SMF are better for PS and DS pixels' optimization, respectively. The SMF-POLOPT, which combines the merits of SMF and PolSAR optimization, is more inclusive for both PS and DS pixels and thus its performance is the best.

\section{DISCUSSION}

To discuss and compare the advantages and shortcomings of all the algorithms included in this paper, their main characteristics and performances on the pixels' density are listed in Table I. The computation time for each algorithm at the phase optimization stage has been also listed in the table. The implementation is in IDL, and the processings have been carried out on a workstation equipped with an 8-core Intel(R) Xeon(R) E5620 processor $(2.4 \mathrm{GHz})$ and $60 \mathrm{~GB}$ of RAM.

Single-pol $D_{A}$ is the simplest approach, neither filtering nor optimization is applied, thus it presents the highest processing efficiency. However, it obtains the lowest pixels' density within the two test areas. The ESM algorithm carries out the PolSAR optimization (POLOPT) by minimizing the quality estimator $D_{A}$, therefore it can improve PS pixels' phase quality and more PS pixels w.r.t. the single-pol $D_{A}$ method can be obtained. Nevertheless, it does not show a significant improvement for DS pixels, as expected. SMF does not modify PS pixels and adaptively filters DS ones, as a result it only increases the number of qualified DS pixels. It is more suitable than ESM in the suburban or barely vegetated areas, but in areas where PS pixels are the majority ESM is better. The SMF-POLOPT is a combination of SMF and POLOPT, therefore it is more inclusive and presents the best performance. On the other hand, the SMF-POLOPT is the most computationally intensive algorithm as Table I shows.

For the SAR data sets with full or dual-polarization channels, it is found that the increase of the pixels' density for the full-polarization case (Barcelona test area) is more significant than that of dual-polarization (Murcia test area) with the advanced algorithms. This difference on performance is more noticeable for the SMF-POLOPT approach.

In practice, one of the above four algorithms can be chosen by considering the available data sets, the characteristics of 


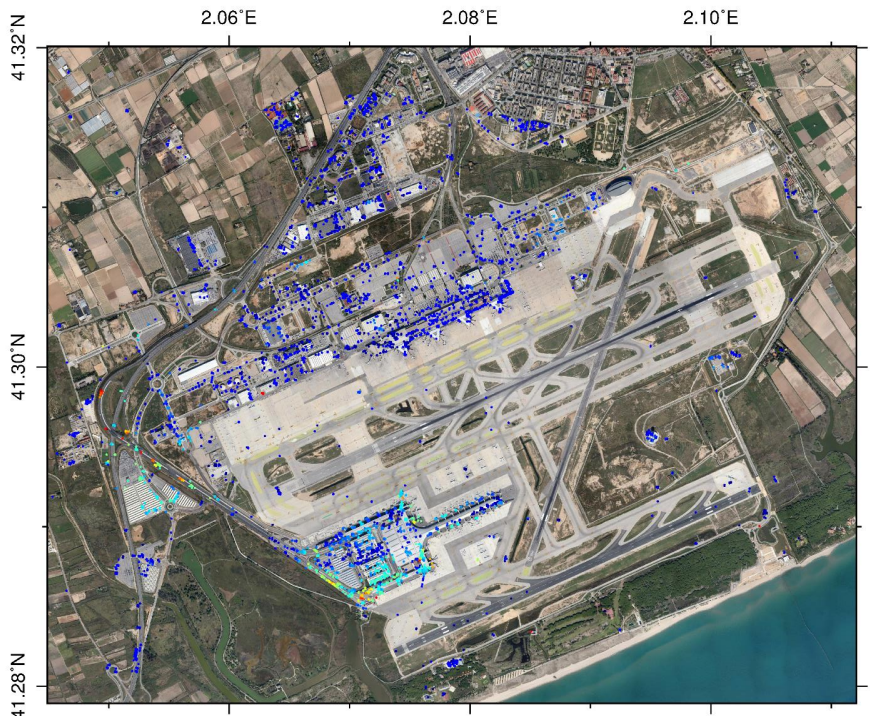

(a) DA HH (4149)

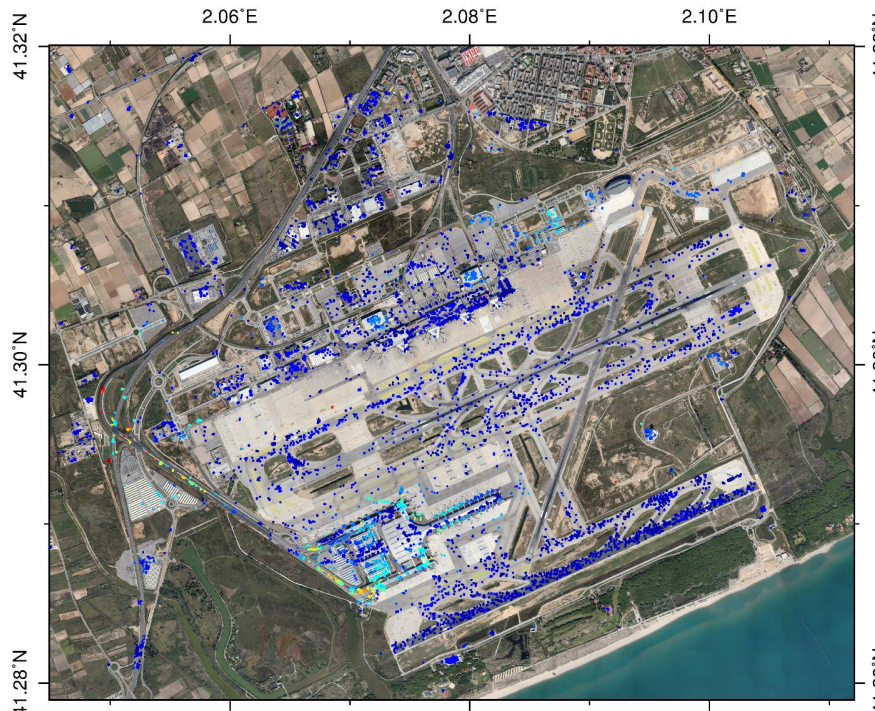

(c) SMF (7502)

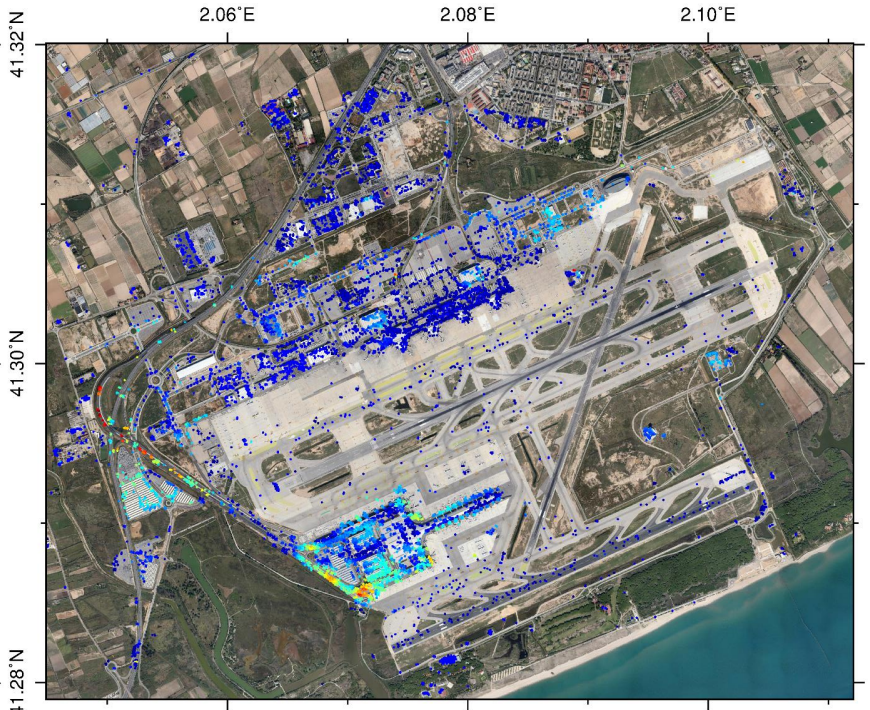

(b) ESM (11248)

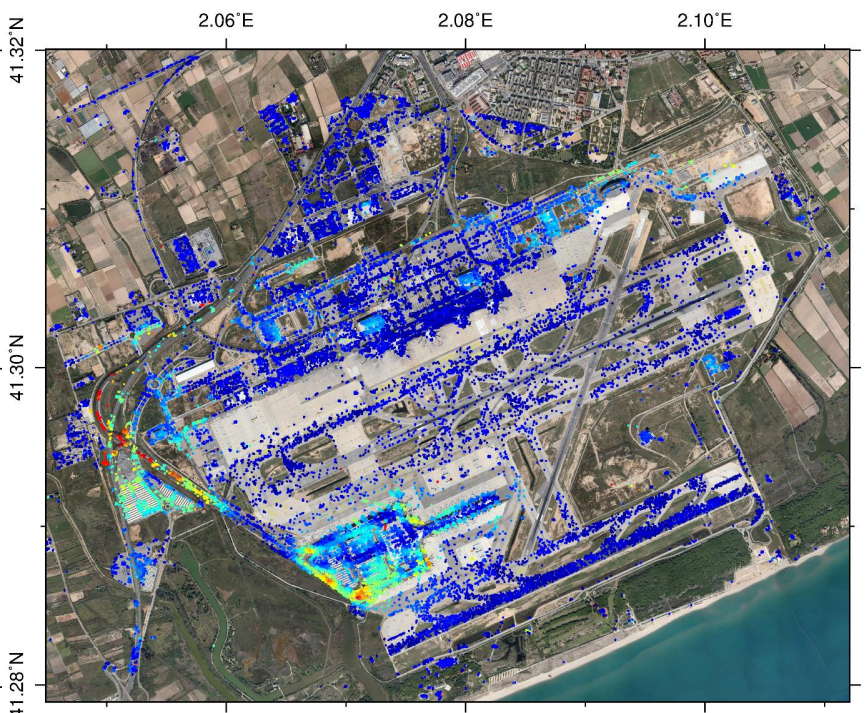

(d) SMF-POLOPT (29963)

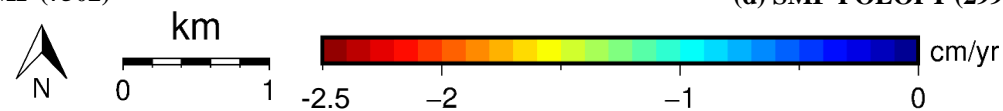

Fig. 7. Barcelona airport ground deformation velocity maps obtained by (a) $D_{A}$, (b) ESM, (c) SMF and (d) SMF-POLOPT. The number in the brackets represents the amount of pixels in each algorithm derived deformation result.

the study area (urban, suburban or rural) and the requirement on data processing efficiency. Generally, the single-pol $D_{A}$ approach is suitable for study areas with abundant PS pixels or areas that are very large and efficient processing is required. The ESM (better for PS pixels' optimization) and SMF (better for DS pixels' filtering) are good replacements of the singlepol $D_{A}$ method when the detailed deformation need to be detected and affordable computation cost can be accepted. If the expensive computation cost can be afforded and higher pixel density is expected, the SMF-POLOPT is the optimal algorithm among the four. It should also be noted that, except for the single-pol $D_{A}$ approach, the other three can be used only when the multi-polarization SAR data sets are available.

\section{CONCLUSION}

In this paper, an adaptive multi-temporal Pol(DIn)SAR filtering and phase optimization algorithm, i.e. SMF-POLOPT, has been proposed. It is inspired by Lee's PolSAR filter [33] and PolDInSAR optimization techniques [15], [32]. This algorithm, which is based on the Scattering-Mechanism based Filtering (SMF) and adaptive POLInSAR OPTimization (POLOPT), can separately filter or (and) optimize PS and DS pixels. Moreover, an inclusive pixel selection method based on pixels' phase standard deviation (STD) has been introduced and developed to identify high quality pixels for PSI processing in this scheme.

Two SAR data sets, full- and dual-polarization, have been used to evaluate the performance of the proposed algorithm. 


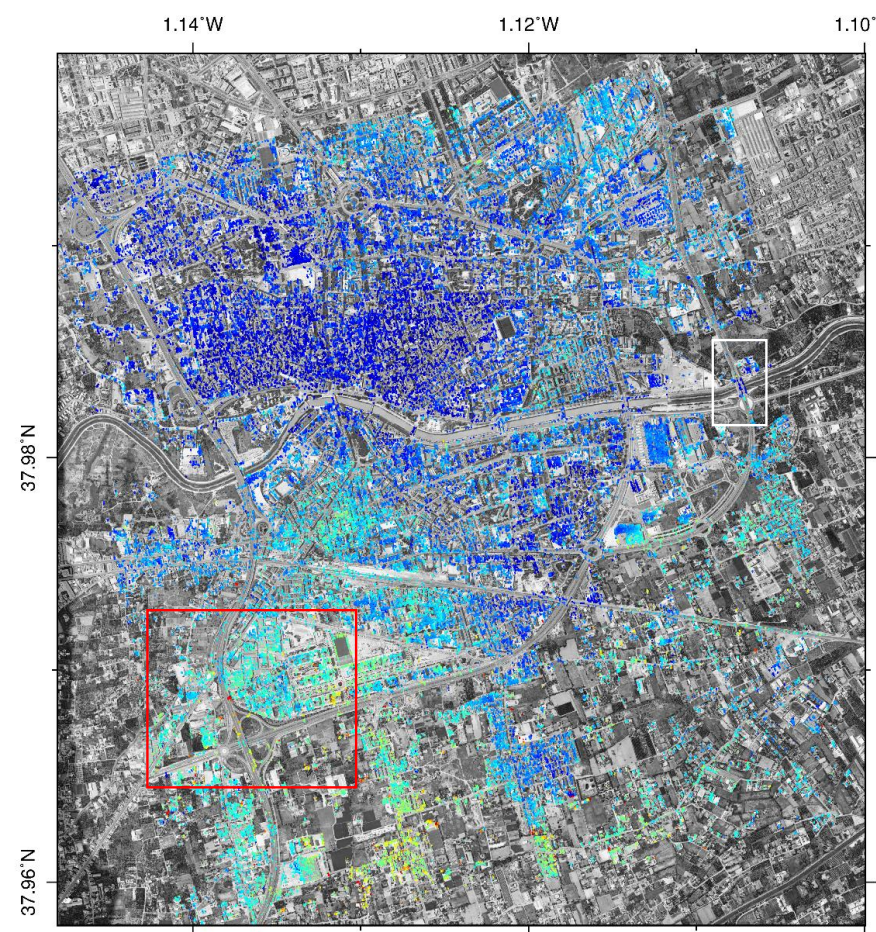

(a) DA HH (162513)

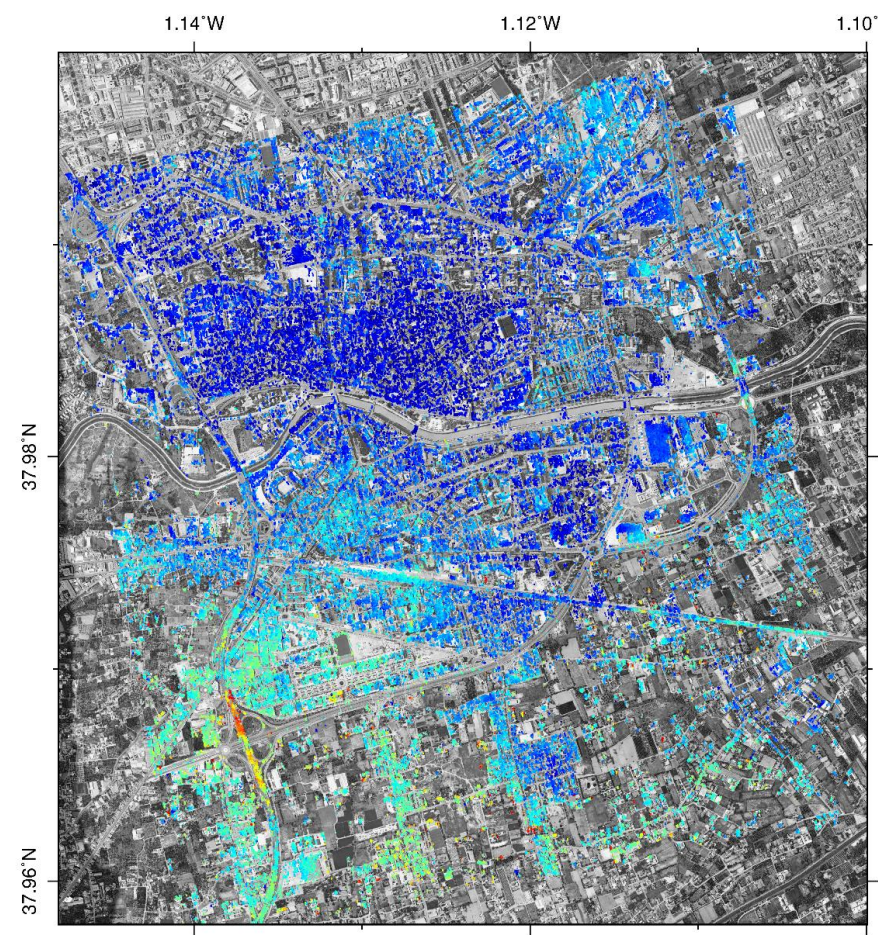

(c) SMF (278522)

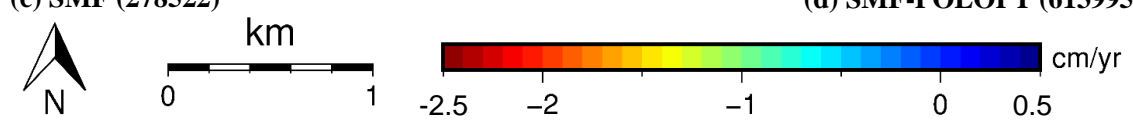

Fig. 8. Murcia ground deformation velocity maps obtained by (a) $D_{A}$, (b) ESM, (c) SMF and (d) SMF-POLOPT. The number in the brackets represents the amount of pixels in each algorithm derived deformation result.

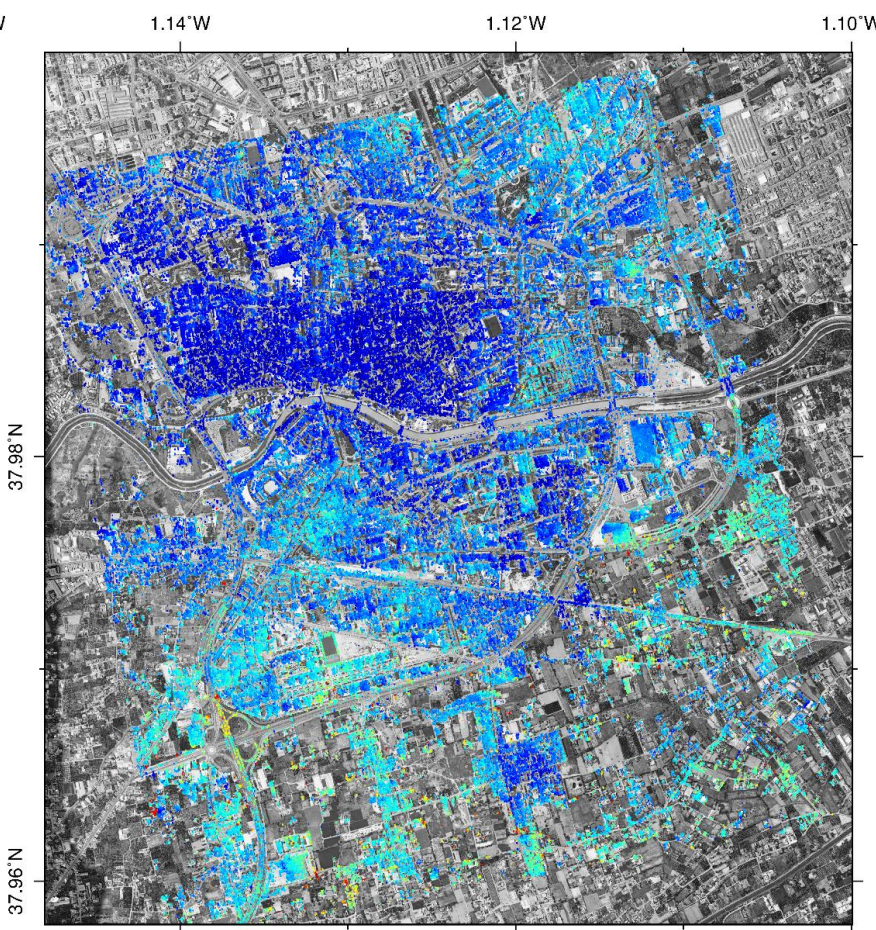

(b) ESM (385407)

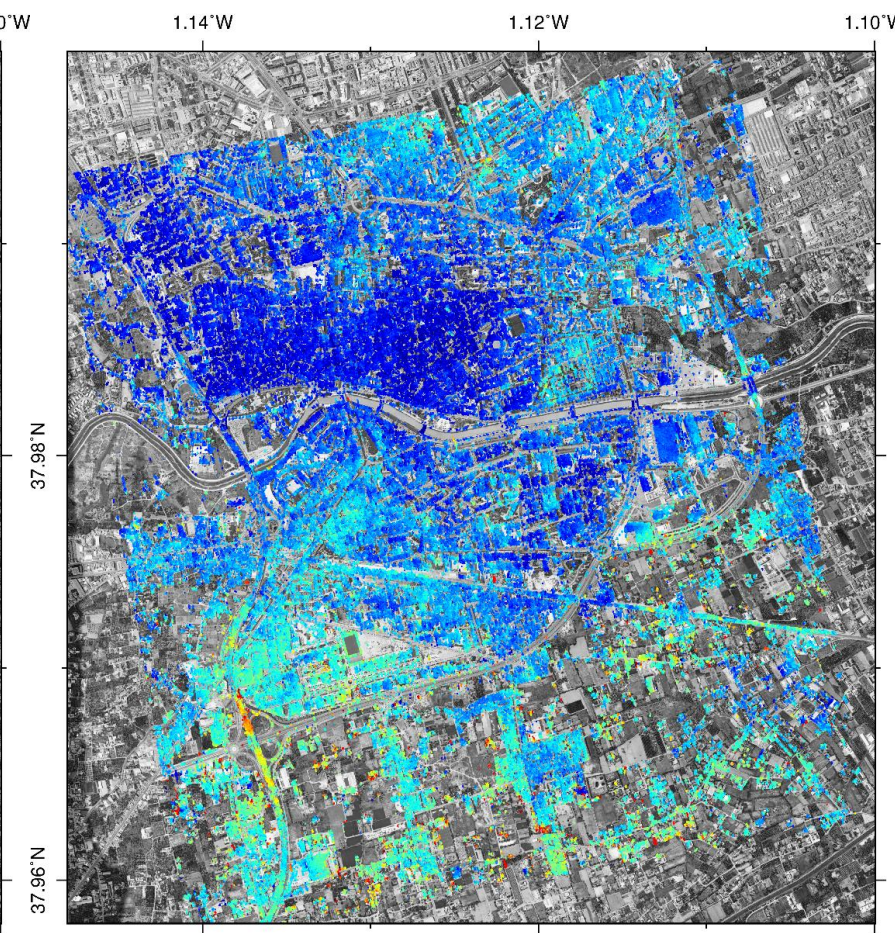

(d) SMF-POLOPT (613995)

$\mathrm{m} / \mathrm{yr}$ 


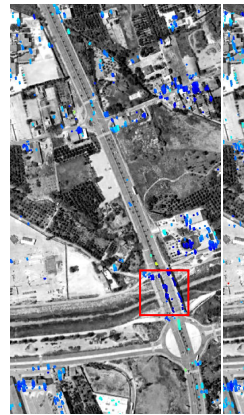

(a) DA HH

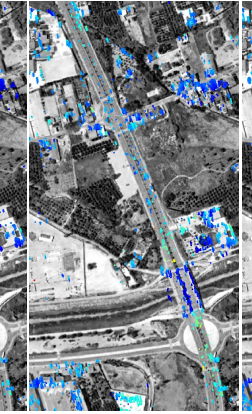

(b) ESM

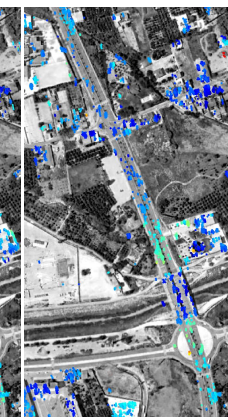

(c) SMF

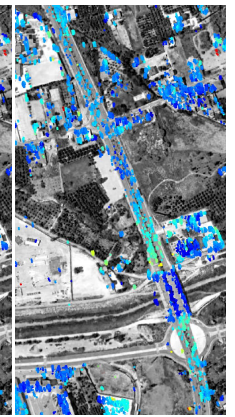

(d) SMF-POLOPT

Fig. 9. The first detailed view of Murcia ground deformation. The same color scale as that in Fig. 8 is used and the red rectangle indicates the location of the stable bridge.

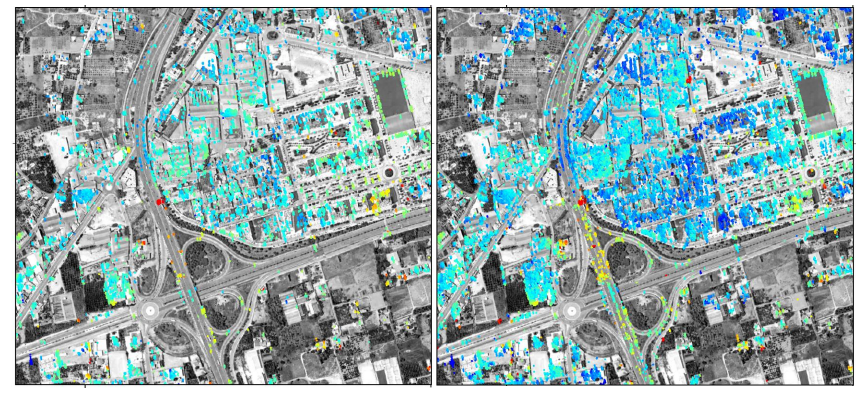

(a) DA HH

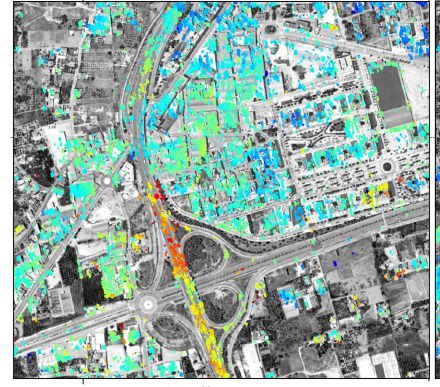

(c) SMF (b) ESM

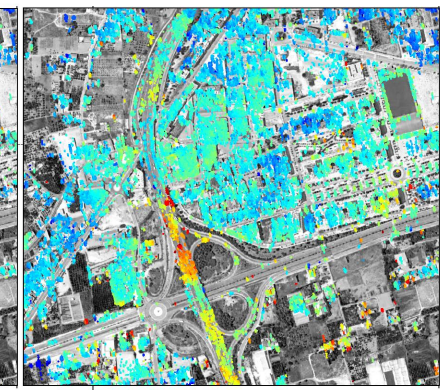

(d) SMF-POLOPT

Fig. 10. The second detailed view of Murcia ground deformation. The same color scale as that in Fig. 8 is used.

For PolSAR filtering, the SMF achieves the speckle reduction and details preservation simultaneously, and it outperforms the other two well established PolSAR filters [33], [41]. In terms of PolDInSAR phase optimization, the proposed SMFPOLOPT presents better performance than the other algorithms. In both test areas, significant improvements regarding pixels' densities have been achieved by the SMF-POLOPT algorithm. Particularly, an increase of around $\times 7.2$ and $\times 3.8$ in the number of pixels w.r.t. the single-pol $D_{A}$ method have been achieved with the SMF-POLOPT approach with Barcelona (full-polarization) and Murcia (dual-polarization) SAR data sets, respectively. The corresponding improvements achieved by the ESM and SMF algorithms are $\times 2.7$ and $\times 1.8$ for the full-polarization case, $\times 2.4$ and $\times 1.7$ for the dual-polarization case w.r.t. the single-pol $D_{A}$ method, respectively.

The advantages and shortcomings of the four employed ground deformation monitoring algorithms have been dis-

TABLE I

COMPARISON BETWEEN DIFFERENT GROUND DEFORMATION ESTIMATION ALGORITHMS

\begin{tabular}{lcccc}
\hline \hline Method & SMF & OPT & Barcelona & Murcia \\
\hline$D_{A}$ & No & No & $\times 1.0(0 \mathrm{~h})$ & $\times 1.0(0 \mathrm{~h})$ \\
ESM & No & Yes & $\times 2.7(3.8 \mathrm{~h})$ & $\times 2.4(4.2 \mathrm{~h})$ \\
SMF & Yes & No & $\times 1.8(1.0 \mathrm{~h})$ & $\times 1.7(4.7 \mathrm{~h})$ \\
SMF-POLOPT & Yes & Yes & $\times 7.2(4.9 \mathrm{~h})$ & $\times 3.8(13.3 \mathrm{~h})$ \\
\hline
\end{tabular}

Barcelona and Murcia columns give out the multiples of the numbers of the obtained pixels by advanced algorithms w.r.t. that by the single-pol $D_{A}$ method at these two test sites, respectively. The time in the brackets are the corresponding computation time of optimization stage for each case. OPT means the polarimetric OPTimization processing.

cussed and analyzed. The proposed SMF method can adaptive filter DS pixels and preserves PS ones, it is thus a good alternative to the ESM approach that mainly optimizes PS pixels. When an expensive computation cost is acceptable, SMF-POLOPT is the best one among the four methods for both dual- and full-pol SAR data. The proposed adaptive filtering and phase optimization algorithm, i.e. SMF-POLOPT, can be used for adaptive Pol(DIn)SAR images' filtering or interferograms' polarimetric optimization when multi-temporal PolSAR images are available.

There are still open issues to investigate. For instance, other advanced PolSAR filters can be evaluated to find the one with better performances but keeping in mind that, being the objective a PSI processing involving large numbers of images, their computational efficiency is a key factor. Similarly, the rigorous evaluation of such filters would require a quantitative evaluation through their performance on bias reduction, mean preservation and ENL.

\section{ACKNOWLEDGMENT}

The Radarsat- 2 images were provided by MDA in the framework of the scientific project SOAR-EU 6779. The TerraSAR-X images have been provided by the German Aerospace Center (DLR) in the framework of Project GEO0389 of the TerraSAR-X scientific program. The DEMs were from the Instituto Geografico Nacional of Spain. Some figures were prepared using the public domain GMT software (Wessel and Smith, 1998). Optical images provided by GoogleEarth.

\section{REFERENCES}

[1] A. Ferretti, C. Prati, and F. Rocca, "Permanent scatterers in SAR interferometry," IEEE Transactions on geoscience and remote sensing, vol. 39, no. 1, pp. 8-20, 2001.

[2] _ _ "Nonlinear subsidence rate estimation using permanent scatterers in differential SAR interferometry," IEEE Transactions on geoscience and remote sensing, vol. 38, no. 5, pp. 2202-2212, 2000.

[3] A. Hooper, H. Zebker, P. Segall, and B. Kampes, "A new method for measuring deformation on volcanoes and other natural terrains using InSAR persistent scatterers," Geophysical research letters, vol. 31, no. 23, 2004.

[4] A. Hooper, P. Segall, and H. Zebker, "Persistent scatterer interferometric synthetic aperture radar for crustal deformation analysis, with application to volcán alcedo, galápagos," Journal of Geophysical Research: Solid Earth, vol. 112, no. B7, 2007.

[5] P. Berardino, G. Fornaro, R. Lanari, and E. Sansosti, "A new algorithm for surface deformation monitoring based on small baseline differential SAR interferograms," IEEE Transactions on Geoscience and Remote Sensing, vol. 40, no. 11, pp. 2375-2383, 2002. 
[6] O. Mora, J. J. Mallorqui, and A. Broquetas, "Linear and nonlinear terrain deformation maps from a reduced set of interferometric SAR images," IEEE Transactions on Geoscience and Remote Sensing, vol. 41, no. 10, pp. 2243-2253, 2003.

[7] P. Blanco-Sanchez, J. J. Mallorquí, S. Duque, and D. Monells, "The coherent pixels technique (CPT): An advanced DInSAR technique for nonlinear deformation monitoring," Pure and Applied Geophysics, vol. 165 , no. 6, pp. 1167-1193, 2008.

[8] A. Ferretti, A. Fumagalli, F. Novali, C. Prati, F. Rocca, and A. Rucci, "A new algorithm for processing interferometric data-stacks: SqueeSAR," IEEE Transactions on Geoscience and Remote Sensing, vol. 49, no. 9, pp. 3460-3470, 2011

[9] Y. Wang, X. Zhu, and R. Bamler, "Retrieval of phase history parameters from distributed scatterers in urban areas using very high resolution SAR data," ISPRS Journal of Photogrammetry and Remote Sensing, vol. 73, pp. pp-89, 2012.

[10] A. Parizzi and R. Brcic, "Adaptive InSAR stack multilooking exploiting amplitude statistics: A comparison between different techniques and practical results," IEEE Geoscience and Remote Sensing Letters, vol. 8, no. 3, pp. 441-445, 2011.

[11] G. Fornaro, S. Verde, D. Reale, and A. Pauciullo, "CAESAR: An approach based on covariance matrix decomposition to improve multibaseline-multitemporal interferometric SAR processing," IEEE Transactions on Geoscience and Remote Sensing, vol. 53, no. 4, pp. 2050-2065, 2015.

[12] L. Pipia, X. Fabregas, A. Aguasca, C. Lopez-Martinez, S. Duque, J. J. Mallorqui, and J. Marturia, "Polarimetric differential SAR interferometry: First results with ground-based measurements," IEEE Geoscience and Remote Sensing Letters, vol. 6, no. 1, pp. 167-171, 2009.

[13] V. D. Navarro-Sanchez, J. M. Lopez-Sanchez, and F. Vicente-Guijalba, "A contribution of polarimetry to satellite differential SAR interferometry: Increasing the number of pixel candidates," IEEE Geoscience and Remote Sensing Letters, vol. 7, no. 2, pp. 276-280, 2010.

[14] V. D. Navarro-Sanchez, J. M. Lopez-Sanchez, and L. Ferro-Famil, "Polarimetric approaches for persistent scatterers interferometry," IEEE Transactions on Geoscience and Remote Sensing, vol. 52, no. 3, pp. 1667-1676, 2014.

[15] R. Iglesias, D. Monells, X. Fabregas, J. J. Mallorqui, A. Aguasca, and C. Lopez-Martinez, "Phase quality optimization in polarimetric differential SAR interferometry," IEEE transactions on geoscience and remote sensing, vol. 52, no. 5, pp. 2875-2888, 2014.

[16] D. Monells, R. Iglesias, J. J. Mallorquí, X. Fàbregas, and C. LopezMartinez, "Phase quality optimization in orbital differential SAR interferometry with fully polarimetric data," in Geoscience and Remote Sensing Symposium (IGARSS), 2012 IEEE International. IEEE, 2012, pp. 1864-1867.

[17] R. Iglesias, X. Fabregas, A. Aguasca, C. López-Martínez, and A. Alonso-González, "Advanced polarimetric optimization for DInSAR applications with ground-based SAR," in Synthetic Aperture Radar, 2012. EUSAR. 9th European Conference on. VDE, 2012, pp. 513516.

[18] V. D. Navarro-Sanchez and J. M. Lopez-Sanchez, "Improvement of persistent-scatterer interferometry performance by means of a polarimetric optimization," IEEE Geoscience and Remote Sensing Letters, vol. 9, no. 4, pp. 609-613, 2012.

[19] R. Iglesias, D. Monells, X. Fabregas, J. J. Mallorqui, A. Aguasca, and C. Lopez-Martínez, "Phase quality optimization techniques and limitations in polarimetric differential SAR interferometry," transformation, vol. 17, p. 18, 2013.

[20] B. Wu, L. Tong, Y. Chen, and L. He, "New methods in multibaseline polarimetric SAR interferometry coherence optimization," IEEE Geoscience and Remote Sensing Letters, vol. 12, no. 10, pp. 2016-2020, 2015.

[21] — "Improved SNR optimum method in PolDInSAR coherence optimization," IEEE Geoscience and Remote Sensing Letters, vol. 13, no. 7, pp. 982-986, 2016.

[22] Z. Sadeghi, M. J. V. Zoej, and J.-P. Muller, "Monitoring land subsidence in a rural area using a combination of ADInSAR and polarimetric coherence optimization," IEEE Journal of Selected Topics in Applied Earth Observations and Remote Sensing, vol. 10, no. 8, pp. 3582-3590, 2017.

[23] M. Esmaeili and M. Motagh, "Improved persistent scatterer analysis using amplitude dispersion index optimization of dual polarimetry data," ISPRS Journal of Photogrammetry and Remote Sensing, vol. 117, pp. 108-114, 2016.

[24] M. Esmaeili, M. Motagh, and A. Hooper, "Application of dualpolarimetry SAR images in multitemporal InSAR processing," IEEE
Geoscience and Remote Sensing Letters, vol. 14, no. 9, pp. 1489-1493, 2017.

[25] K. Ishitsuka, T. Matsuoka, and M. Tamura, "Persistent scatterer selection incorporating polarimetric SAR interferograms based on maximum likelihood theory," IEEE Transactions on Geoscience and Remote Sensing, vol. 55, no. 1, pp. 38-50, 2017.

[26] A. G. Mullissa, V. Tolpekin, A. Stein, and D. Perissin, "Polarimetric differential SAR interferometry in an arid natural environment," International journal of applied earth observation and geoinformation, vol. 59, pp. $9-18,2017$.

[27] A. G. Mullissa, D. Perissin, V. A. Tolpekin, and A. Stein, "Polarimetrybased distributed scatterer processing method for PSI applications," IEEE transactions on geoscience and remote sensing, vol. 56, no. 6 , pp. 3371-3382, 2018.

[28] Z. Sadeghi, M. J. V. Zoej, and J.-P. Muller, "Combination of persistent scatterer interferometry and single-baseline polarimetric coherence optimisation to estimate deformation rates with application to tehran basin," PFG-Journal of Photogrammetry, Remote Sensing and Geoinformation Science, vol. 85, no. 5, pp. 327-340, 2017.

[29] R. Iglesias, J. J. Mallorqui, and P. López-Dekker, "DInSAR pixel selection based on sublook spectral correlation along time." IEEE Trans. Geoscience and Remote Sensing, vol. 52, no. 7, pp. 3788-3799, 2014.

[30] R. Iglesias, D. Monells, C. López-Martínez, J. J. Mallorqui, X. Fabregas, and A. Aguasca, "Polarimetric optimization of temporal sublook coherence for DInSAR applications," IEEE Geoscience and Remote Sensing Letters, vol. 12, no. 1, pp. 87-91, 2015.

[31] Z. Sadeghi, M. J. V. Zoej, A. Hooper, and J. M. Lopez-Sanchez, "A new polarimetric persistent scatterer interferometry method using temporal coherence optimization," IEEE Transactions on Geoscience and Remote Sensing, no. 99, pp. 1-9, 2018.

[32] V. D. Navarro-Sanchez and J. M. Lopez-Sanchez, "Spatial adaptive speckle filtering driven by temporal polarimetric statistics and its application to PSI," IEEE Transactions on Geoscience and Remote Sensing, vol. 52, no. 8, pp. 4548-4557, 2014.

[33] J.-S. Lee, M. R. Grunes, D. L. Schuler, E. Pottier, and L. Ferro-Famil, "Scattering-model-based speckle filtering of polarimetric SAR data," IEEE Transactions on Geoscience and Remote Sensing, vol. 44, no. 1, pp. 176-187, 2006

[34] J.-S. Lee, M. R. Grunes, and G. De Grandi, "Polarimetric SAR speckle filtering and its implication for classification," IEEE Transactions on Geoscience and remote sensing, vol. 37, no. 5, pp. 2363-2373, 1999.

[35] J.-S. Lee, T. L. Ainsworth, Y. Wang, and K.-S. Chen, "Polarimetric SAR speckle filtering and the extended sigma filter," IEEE Transactions on geoscience and remote sensing, vol. 53, no. 3, pp. 1150-1160, 2015.

[36] J.-S. Lee, M. R. Grunes, T. L. Ainsworth, L.-J. Du, D. L. Schuler, and S. R. Cloude, "Unsupervised classification using polarimetric decomposition and the complex Wishart classifier," IEEE Transactions on Geoscience and Remote Sensing, vol. 37, no. 5, pp. 2249-2258, 1999.

[37] E. Pottier and J.-S. Lee, "Application of the H/A/alpha polarimetric decomposition theorem for unsupervised classification of fully polarimetric SAR data based on the wishart distribution," in SAR workshop: CEOS Committee on Earth Observation Satellites, vol. 450, 2000, p. 335.

[38] L. Ferro-Famil, E. Pottier, and J.-S. Lee, "Unsupervised classification of multifrequency and fully polarimetric SAR images based on the H/A/Alpha-Wishart classifier," IEEE Transactions on Geoscience and Remote Sensing, vol. 39, no. 11, pp. 2332-2342, 2001.

[39] J.-S. Lee and E. Pottier, Polarimetric radar imaging: from basics to applications. CRC press, 2009.

[40] S. Samsonov and K. Tiampo, "Polarization phase difference analysis for selection of persistent scatterers in SAR interferometry," IEEE Geoscience and Remote Sensing Letters, vol. 8, no. 2, pp. 331-335, 2011.

[41] G. Vasile, E. Trouvé, J.-S. Lee, and V. Buzuloiu, "Intensity-driven adaptive-neighborhood technique for polarimetric and interferometric SAR parameters estimation," IEEE Transactions on Geoscience and Remote Sensing, vol. 44, no. 6, pp. 1609-1621, 2006.

[42] S. R. Cloude and K. P. Papathanassiou, "Polarimetric SAR interferometry," IEEE Transactions on Geoscience and Remote Sensing, vol. 36, no. 5, pp. 1551-1565, 1998.

[43] J.-S. Lee, K. W. Hoppel, S. A. Mango, and A. R. Miller, "Intensity and phase statistics of multilook polarimetric and interferometric SAR imagery," IEEE Transactions on Geoscience and Remote Sensing, vol. 32, no. 5, pp. 1017-1028, 1994.

[44] R. F. Hanssen, Radar interferometry: data interpretation and error analysis. Springer Science \& Business Media, 2001, vol. 2.

[45] C.-A. Deledalle, L. Denis, F. Tupin, A. Reigber, and M. Jäger, "NL-SAR: A unified nonlocal framework for resolution-preserving (Pol)(In)SAR 
denoising," IEEE Transactions on Geoscience and Remote Sensing, vol. 53, no. 4, pp. 2021-2038, 2015.

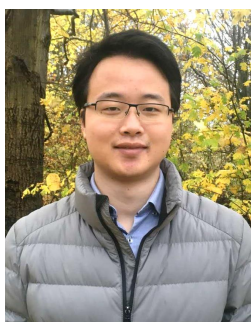

Feng Zhao (S'17) received the M.S. degrees in geodesy and surveying engineering from China University of Mining and Technology (CUMT), Xuzhou, China, in 2016. He is currently working toward the Ph.D. degree at the Universitat Politècnica de Catalunya, Barcelona, Spain.

He joined the CommSensLab, Universitat Politècnica de Catalunya (UPC), in September 2016, where his main work focuses on the development of advanced pixel selection and optimization algorithms for multi-temporal (Pol)DInSAR techniques. His research interests include advanced DInSAR techniques and its application on terrain deformation detection.

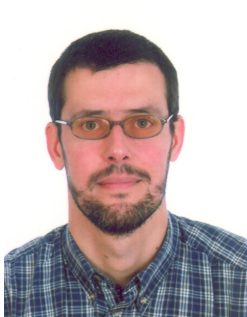

Jordi J. Mallorqui (S'93-M'96-SM'13) was born in Tarragona, Spain, in 1966. He received the Ingeniero degree in telecommunications engineering and the Doctor Ingeniero degree in telecommunications engineering for his research on microwave tomography for biomedical applications in the Department of Signal Theory and Communications from the Universitat Polit'ecnica de Catalunya (UPC), Barcelona, Spain, in 1990 and 1995, respectively. Since 1993, he has been teaching at the School of Telecommunications Engineering of Barcelona, UPC, first as an Assistant Professor, later in 1997 as an Associate Professor, and since 2011 as a Full Professor. Since October 2017 he is the Director of the Department of Signal Theory and Communications (UPC).

His teaching activity involves microwaves, radionavigation systems, and remote sensing. He spent a sabbatical year with the Jet Propulsion Laboratory, Pasadena, CA, USA, in 1999, working on interferometric airborne synthetic aperture radar (SAR) calibration algorithms. He is currently working on the application of SAR interferometry to terrain deformation monitoring with orbital, airborne, and ground data; vessel detection and classification from SAR images; and 3-D electromagnetic (EM) simulation of SAR systems. He is also collaborating in the design and construction of a ground-based SAR interferometer for landslide control. He has published more than 100 papers on microwave tomography, EM numerical simulation, SAR processing, interferometry, and differential interferometry in refereed journals and international symposia. 